$8^{\mathrm{m}} \cdot 5$ und $\beta=\mathrm{BD}+0^{\circ} 4022,9^{\mathrm{m}} \cdot 3$ photometrisch geschätzt und dabei gefunden:

kontinuierliches Spektrum: $\alpha{ }_{4} N \circ \beta$

also Sterngröße des kontinuierlichen Spektrums der Nova:

$$
N=9^{\mathrm{m}} \cdot 30 \text {. }
$$

Andererseits ergab die direkte Schätzung der drei Sterne ohne das Spektroskop: $N$ I $\alpha 5 \beta$

also die Gesamthelligkeit der Nova:

$$
N=8^{m} \cdot 34 \text {. }
$$

Hieraus ergibt sich die Sterngröße $x$ des gasförmigen Teils des Sterns gemäß der Gleichung:

während für die Sterngröße der glühflüssigen Komponente der Nova der Wert $y=9^{\mathrm{m}} \cdot 3^{\circ}$

nach oben anzusetzen ist. Der gasförmige Teil ist also an sich etwa eine halbe Größenklasse heller als der glühflüssige Teil des Sterns.

Es wurden folgende gewöhnliche Schätzungen erhalten:

$$
\begin{array}{rlll}
\text { I9 19 April 2 I } & N \text { I2 } \text { I }_{4} \beta & N=6^{\mathrm{m}} 5^{8} \\
\text { Okt. I9 } & N \text { I } \alpha 5 \beta & N=8.34 \\
\text { 1920 Mai 24 } & \alpha 3 N_{4} \beta & N=8.84 .
\end{array}
$$

Stockholm, Sternwarte, I 920 Sept. 28.
3. Nova Lyrae (r.9.9)

$\alpha \mathrm{I}_{900.0}={ }_{1} 8^{\mathrm{h}} 49^{\mathrm{m}} 30^{\mathrm{s}} \cdot \delta \mathrm{I}_{900.0}=+29^{\circ} 6^{\prime}$.

Ich habe am I5. Sept. I 920 diesen Neuen Stern mit dem Zöllnerschen Spektroskope angesehen. Bei der Schwäche des Objektes - es war etwa Io-I I. Größe - erschien das Spektrum völlig monochromatisch. Jedoch ist das Bild des Sterns im Spektroskope etwas heller als ohne dasselbe, wofür wohl anzunehmen ist, daß der Lichtknoten ausgedehnt bzw. wahrscheinlich doppelt ist. Es wurde visuell geschätzt: $\alpha_{4} N$ I $\beta$.

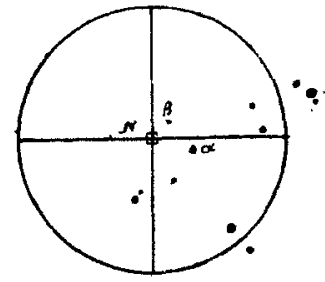

Zur Indentifizierung dieser '́ंergleichsterne, die nicht in der B. D. enthalten sind, füge ich die nebenstehende Skizze der Umgebung der Nova Lyrae (I9r9) bei, die nach einer der aufgenommenen Platten beiläufig angefertigt ist.

Die Darstellung ist diejenige des umkehrenden Fernrohrs. Der Durehmesser des Gesichtsfeldes mißt ungefähr $20^{\prime}$.

\begin{tabular}{|c|c|c|c|c|c|c|c|c|c|c|c|c|c|c|c|}
\hline \multicolumn{3}{|c|}{ Bezeichnung } & \multicolumn{3}{|c|}{$\begin{array}{c}\alpha_{1} 875 \\
20^{\mathrm{h}} 29^{\mathrm{m}} 16^{\mathrm{s}}: 4\end{array}$} & \multicolumn{3}{|c|}{$\delta 1875$} & \multicolumn{3}{|c|}{$\begin{array}{c}\alpha, 855 \\
20^{\text {h }} 28^{m} 45^{s}\end{array}$} & \multicolumn{2}{|c|}{$\begin{array}{r}81855 \\
+54^{\circ} 27^{\prime} 6\end{array}$} & \multirow{2}{*}{$\begin{array}{l}19.80 \\
10.5 \\
\text { I I }\end{array}$} & \multirow{2}{*}{$\begin{array}{l}05 \cdot 42 \\
\mathrm{I} 3^{\mathrm{m}} \cdot 5 \\
\mathrm{I} 2\end{array}$} \\
\hline$b$ & 80.1920 & Cygni & 20 & 29 & 22.9 & 53 & $5^{I}$ & 45 & 20 & 28 & $5^{I}$ & 53 & 47.7 & & \\
\hline$c$ & $8 \mathrm{I.1920}$ & 》 & 20 & $3 \mathbf{I}$ & 34.2 & $5^{8}$ & 20 & 3 & 20 & $3 I$ & 7 & $5^{8}$ & I 6.0 & I 4 & I 2 \\
\hline$d$ & 82.1920 & \# & 20 & $3^{2}$ & I 3.4 & 60 & 53 & 39 & 20 & $3 I$ & 51 & 60 & 49.5 & ${ }^{1} 3$ & I 2 \\
\hline$?$ & 83.1920 & $\gg$ & 20 & $3^{2}$ & 57.1 & 54 & I 3 & I 2 & 20 & 32 & 25 & 54 & 9. I & $=16$ & 14 \\
\hline & 84.1920 & Cephei & 20 & 36 & I 8.9 & 59 & 5 & 54 & 20 & 35 & $5^{r}$ & 59 & r. 7 & I 3.5 & I 2.5 \\
\hline$g$ & 920 & Cygni & 20 & 44 & 15.6 & 53 & 34 & 59 & 20 & 43 & $4 I$ & 53 & 30.6 & $<16$ & I I \\
\hline$h$ & 86.1920 & Cephèi & 20 & 48 & $35 \cdot 3$ & 55 & 56 & 54 & 20 & 48 & 3 & 55 & 52.4 & I 6 & I 4 \\
\hline 8 & 87.1920 & 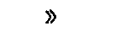 & 20 & $5^{6}$ & I 4.5 & $5^{8}$ & 54 & $2 \mathrm{I}$ & 20 & 55 & 45 & 58 & 49.7 & I 4 & I 2.5 \\
\hline$k$ & 88.1920 & $»$ & $2 \mathrm{I}$ & o & I $9 \cdot 3$ & 54 . & $5^{8}$ & 8 & 20 & 59 & 45 & 54 & 53.4 & $<16$ & 15 \\
\hline 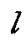 & 89.1920 & $\triangleright$ & $2 \mathrm{I}$ & 5 & 2.2 & 58 & 15 & 26 & $2 \mathrm{I}$ & 4 & 31 & 58 & I 0.6 & 16.5 & I 2 \\
\hline
\end{tabular}
Die Sterne rechts unten sind $\mathrm{BD}+29^{\circ} 33^{8} \mathrm{I}, 33^{82}, 33^{8}$; $9^{\mathrm{m}} \cdot 3,9^{\mathrm{m}} \cdot 0,8^{\mathrm{m}} \cdot 9$.

\section{K. Bohlin.}

\title{
Einige Veränderliche in Cepheus und Cygnus.
}

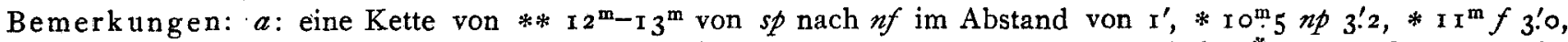

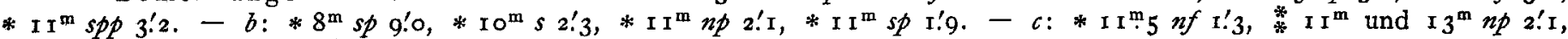

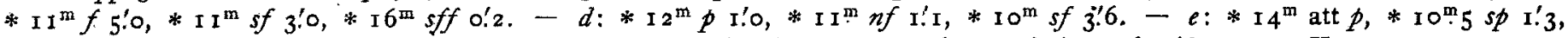

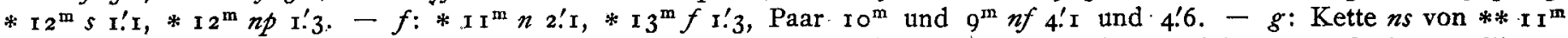

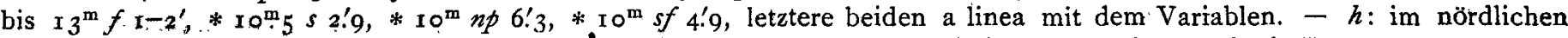

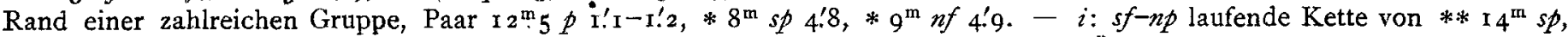



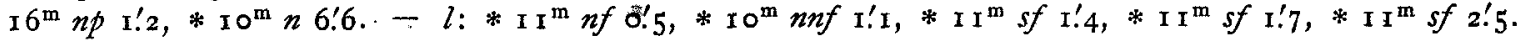

Königstuhl, Ig20 Sept. 23 .

M. Wolf.

Barnards Stern. Zu einer Untersuchung über die Bewegung dieses Schnelläufers benötige ich möglichst viele, über den ganzen Zeitraum seit 1888 (erste Aufnahme von Pickering) sich verteilende Positionen und bitte alle, die dazu in der Lage sind, mir solche mitzuteilen, möglichst unter Angabe der Vergleichsterne.

H. Kienle, München 27, Sternwarte.

Mondbeobachtungen. Zum Zwecke einer Untersuchung über die Bewegung des Mondes im Anschluß an die Beobachtungen der letzten $3^{\circ}$ Jahre wäre die Bekanntgabe etwa noch vorhandener Beobachtungsreihen aus diesem Zeitraum oder die unmittelbare Mitteilung der Resultate an den Unterzeichneten erwünscht.

$K$. Stumpff, stud.-math. et astr., Göttingen, Herzberger Landstraße I I 7 . 